\title{
7-Tesla Functional Cardiovascular MR Using Vectorcardiographic Triggering-Overcoming the Magnetohydrodynamic Effect
}

\author{
Christian Hamilton-Craig ${ }^{1, *}$, Daniel Stäeb ${ }^{1,2}$, Aiman Al Najjar ${ }^{1}$, Kieran O'Brien ${ }^{2}$, William Crawford ${ }^{1,3,4}$, \\ Sabine Fletcher ${ }^{1}$, Markus Barth ${ }^{1}$ (D) and Graham Galloway ${ }^{1,5}$ \\ 1 The Centre for Advanced Imaging, The University of Queensland, Brisbane, QLD 4000, Australia; \\ daniel.staeb@siemens-healthineers.com (D.S.); aiman.alnajjar@cai.uq.edu.au (A.A.N.); \\ william.crawford11@gmail.com (W.C.); sabine.fletcher01@gmail.com (S.F.); m.barth@uq.edu.au (M.B.); \\ graham.galloway@tri.edu.au (G.G.) \\ 2 MR Research Collaborations, Siemens Healthineers Pty Ltd., Bayswater, VIC 3153, Australia; \\ kieran.obrien@siemens-healthineers.com \\ 3 Department of Medical Science, University of Oxford, Oxford 01865, UK \\ 4 School of Information Technology and Electrical Engineering, The University of Queensland, \\ Brisbane, QLD 4000, Australia \\ 5 Imaging Technology, Translational Research Institute, Brisbane, QLD 4000, Australia \\ * Correspondence: c.hamiltoncraig@uq.edu.au
}

\section{check for} updates

Citation: Hamilton-Craig, C.; Stäeb, D.; Al Najjar, A.; O’Brien, K.; Crawford, W.; Fletcher, S.; Barth, M.; Galloway, G. 7-Tesla Functional Cardiovascular MR Using Vectorcardiographic Triggering-Overcoming the Magnetohydrodynamic Effect. Tomography 2021, 7, 323-332. https:// doi.org/10.3390/tomography7030029

Academic Editor: Brian D. Ross

Received: 24 May 2021

Accepted: 19 June 2021

Published: 4 August 2021

Publisher's Note: MDPI stays neutral with regard to jurisdictional claims in published maps and institutional affiliations.

Copyright: (c) 2021 by the authors. Licensee MDPI, Basel, Switzerland. This article is an open access article distributed under the terms and conditions of the Creative Commons Attribution (CC BY) license (https:// creativecommons.org/licenses/by/ $4.0 /)$.

\begin{abstract}
Objective: Ultra-high-field B0 $\geq 7$ tesla (7T) cardiovascular magnetic resonance (CMR) offers increased resolution. However, electrocardiogram (ECG) gating is impacted by the magnetohydrodynamic effect distorting the ECG trace. We explored the technical feasibility of a 7T magnetic resonance scanner using an ECG trigger learning algorithm to quantitatively assess cardiac volumes and vascular flow. Methods: 7T scans were performed on 10 healthy volunteers on a whole-body research MRI MR scanner (Siemens Healthineers, Erlangen, Germany) with 8 channel Tx/32 channels Rx cardiac coils (MRI Tools GmbH, Berlin, Germany). Vectorcardiogram ECG was performed using a learning phase outside of the magnetic field, with a trigger algorithm overcoming severe ECG signal distortions. Vectorcardiograms were quantitatively analyzed for false negative and false positive events. Cine $\mathrm{CMR}$ was performed after 3rd-order $\mathrm{B}_{0}$ shimming using a high-resolution breath-held ECG-retro-gated segmented spoiled gradient echo, and 2D phase contrast flow imaging. Artefacts were assessed using a semi-quantitative scale. Results: 7T CMR scans were acquired in all patients $(100 \%)$ using the vectorcardiogram learning method. 3,142 R-waves were quantitatively analyzed, yielding sensitivity of $97.6 \%$ and specificity of $98.7 \%$. Mean image quality score was 0.9 , sufficient to quantitate both cardiac volumes, ejection fraction, and aortic and pulmonary blood flow. Mean left ventricular ejection fraction was $56.4 \%$, right ventricular ejection fraction was $51.4 \%$. Conclusion: Reliable cardiac ECG triggering is feasible in healthy volunteers at 7T utilizing a stateof-the-art three-lead trigger device despite signal distortion from the magnetohydrodynamic effect. This provides sufficient image quality for quantitative analysis. Other ultra-high-field imaging applications such as human brain functional MRI with physiologic noise correction may benefit from this method of ECG triggering.
\end{abstract}

Keywords: magnetic resonance imaging; MRI scans; cardiology; 7 tesla MRI

\section{Introduction}

Cardiovascular magnetic resonance (CMR) is a increasingly valuable technique for comprehensive morpho-functional evaluation of the left and right ventricles and vascular flow dynamics [1]. Despite their challenges, higher field systems with $\mathrm{B} 0=3 \mathrm{~T}$ are being used in clinical CMR services [2,3]. Ultra-high-field (B0 $\geq 7$ tesla, $7 \mathrm{~T}$ ) magnetic resonance imaging (MRI) offers further advantages of increased resolution, improved signal-to-noise 
ratio (SNR) and potentially improved signal contrast and spatial resolution, but at the disadvantage of increased artefacts and difficulties with ECG-gating [3]. Ultra-high field CMR is challenging due to constraints of energy deposition (specific absorption rate, SAR), transmission field non-uniformity, and B0 magnetic field inhomogeneity [4].

A major challenge for cardiac imaging at ultra-high field strengths is obtaining reliable ECG gating, which is significantly impacted by the magneto-hydrodynamic (MHD) effect distorting the ECG signal $[4,5]$. The interaction of a ferromagnetic conductive fluid (blood) within the static magnetic field $\mathrm{B}_{0}$ induces a voltage perpendicular to both $\mathrm{B}_{0}$ and the direction of blood flow, which is superimposed on the ECG signal, causing substantial derangement of the cutaneous trace [4]. Time-varying magnetic gradient fields also induce voltage perturbations in the ECG leads further distorting the signal [6]. Previous studies of 7T CMR have been often constrained to using pulse oximetry or acoustic triggering $[4,5]$. Vectorcardiography (VCG)-based QRS detection algorithms are commonly employed at 1.5 and $3.0 \mathrm{~T}$, which detect the R-wave peak by recognizing the R-wave's rising amplitude upslope [4,5]. We explored the technical feasibility of a 7T research MRI scanner using a state-of-the-art vector-ECG (VCG) trigger algorithm with a learning phase to create ECG-gated images of left and right ventricles, and aortic and pulmonary vascular flow.

\section{Materials and Methods}

Ultra-high field CMR scans were performed on 10 healthy volunteers using a wholebody 7T research MRI scanner (Siemens Healthineers, Erlangen, Germany) with 8 channel Tx/32 channel Rx cardiac coil (MRI Tools GmbH, Berlin, Germany) under institutional ethics approval (UQ approval 200500050). A detailed discussion of our 7T-CMR acquisition protocol has been described previously [6], but the present data demonstrate application of this technique for quantitative morphofunctional assessment of both left and right ventricular function and flow quantitation. In brief, we used a breath-held, VCG-triggered retrospectively gated two-dimensional spoiled gradient echo FLASH sequence performed after 3rd-order $\mathrm{B}_{0}$ shimming with the following parameters: $\mathrm{FOV}=360 \times 290 \mathrm{~mm}^{2}$, matrix $=352 \times 264$, thickness $=6.0 \mathrm{~mm}, \mathrm{TE}=3.1 \mathrm{~ms}, \mathrm{TR}=63 \mathrm{~ms}$, flip angle $=35^{\circ}$, parallel MRI (GRAPPA), acceleration factor $=2$, reference lines $=24$; phases 20. This allowed cine imaging with in-plane isotropic spatial resolution of $1.0 \mathrm{~mm}$ and a slice thickness of $4.0 \mathrm{~mm}$. Full ventricular coverage was performed with sequential short axis slices from apex to base. Steady-state free precession (SSFP) imaging was not possible due to specific absorption rate concerns (see below). Two-dimensional phase contrast flow images were acquired in the ascending aorta and proximal pulmonary artery positioned at the level of the sino-tubular junctions.

Vectorcardiogram (VCG) based triggering was performed using a three-lead wireless ECG trigger device (Siemens Healthineers GmbH, Erlangen, Germany), in conjunction with a matched filter based VCG trigger algorithm. To improve the synchronization performance, the VCG trigger algorithm was calibrated outside of the magnet bore where the MHD effect is negligible $[5,7,8]$. The learning phase of the algorithm was conducted over a period of at least 30 R-R intervals with the subjects lying on the patient table. Figure 1 shows ECG signals obtained both outside and inside the magnet bore, demonstrating how the trace is substantially altered by the MHD effect causing strong signal distortion. The ST and T-waves are particularly affected, which can lead to incorrect detection of the QRS complex and mis-triggering of the MR image acquisition. A pulse sensor (Siemens Healthineers $\mathrm{GmbH}$, Erlangen, Germany) was attached to the subjects' index finger as a backup trigger device. 

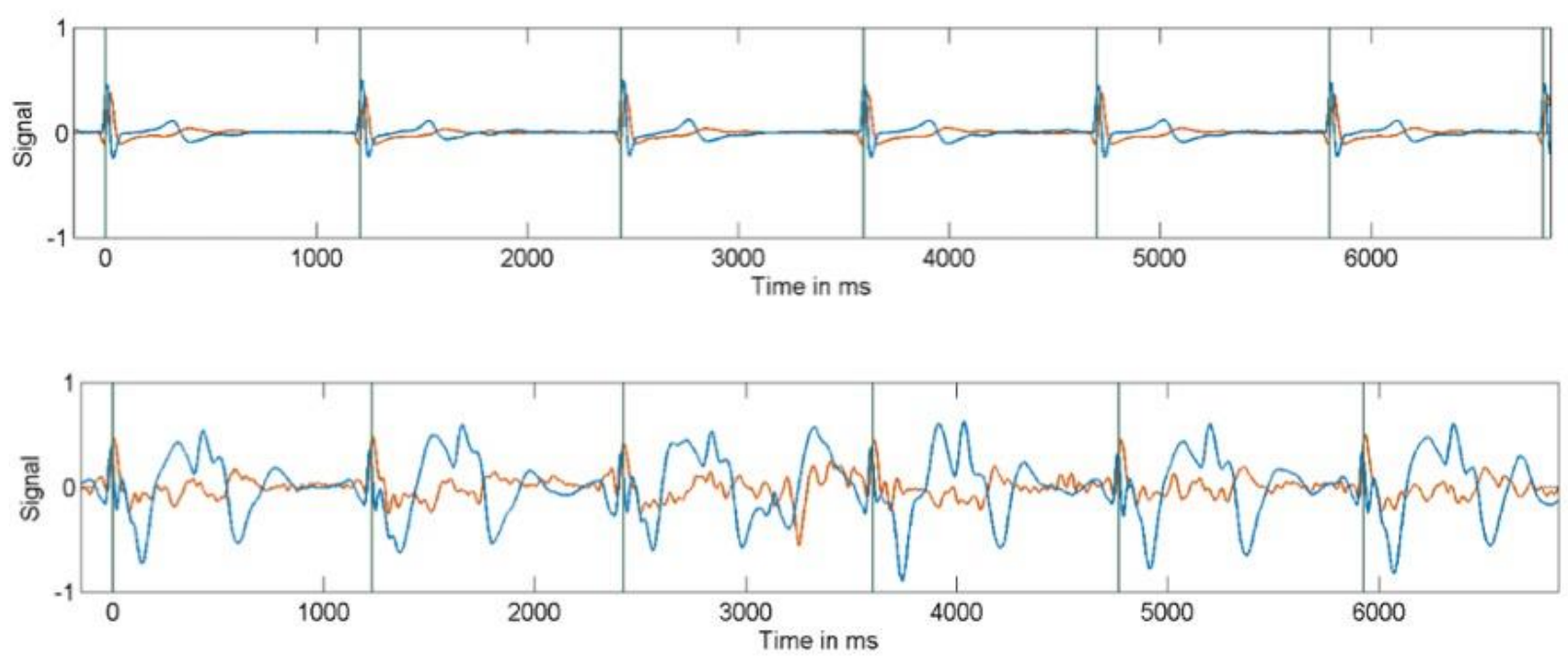

Figure 1. Electrocardiogram (ECG) signals obtained both outside (upper) and inside the magnet bore (lower figure), demonstrating the interference from the magnetic field.

To formally assess the performance of the triggering algorithm, ECG trigger signals and trigger events were recorded. Vectorcardiograms were generated from these data and analyzed quantitatively. To obtain a quantitative estimate, false negative (unidentified $\mathrm{R}$-wave) and false positive (triggered by an event that is not an R-wave) trigger events were identified manually in the ECG recordings. From each subject, a representative continuous section of the ECG signal containing up to 500 trigger events was included in the evaluation. From these results, sensitivity and specificity were calculated as follows:

$$
\begin{aligned}
& \text { Sensitivity }=\left(\mathrm{N}_{\mathrm{RR}}-\mathrm{N}_{\mathrm{FN}}\right) / \mathrm{N}_{\mathrm{RR}} \\
& \text { Specificity }=\left(\mathrm{N}_{\mathrm{RR}}-\mathrm{N}_{\mathrm{FP}}\right) / \mathrm{N}_{\mathrm{RR}}
\end{aligned}
$$

with $\mathrm{N}_{\mathrm{RR}}, \mathrm{N}_{\mathrm{FN}}$ and $\mathrm{N}_{\mathrm{FP}}$ denoting the number of RR-intervals, false negatives and false positives, respectively. A representative section of the recorded VCG curves is shown in Figure 2.
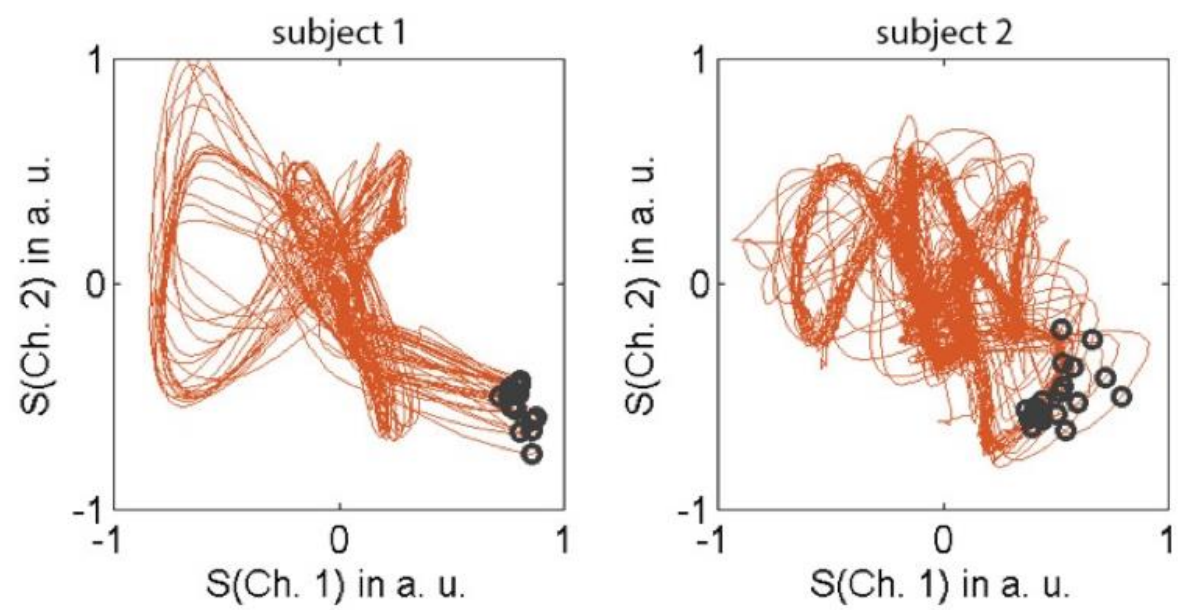

Figure 2. Vectorcardiograms obtained in 2 healthy subjects over a period of $20 \mathrm{~s}$. The trigger events (dark circles) generally occur at a similar location in this vector space.

Volumetric analysis of cine images and flow quantitation from phase images was performed by a European Association of Cardiovascular Imaging level-3 certified expert reader using cmr42 software (v5.9.4, Circle CVi, Calgary, AB, Canada). Rounded ventricular endocardial contours were manually drawn, with the trabeculae and papillary 
muscles included in the blood pool. [9] Phase contrast flow images were analyzed without background correction. The presence of image artefacts was assessed and graded using a semi-quantitative rating scale from 0 (no artefact) to 3 (severe artefact precluding quantitative analysis).

\section{Results}

VCG-gated 7T CMR imaging was successfully performed in $100 \%$ of cases using the learning phase outside of the magnetic field. This resulted in a sufficiently reliable and accurate trigger for CMR acquisition, despite the severe ECG signal distortions from the 7T field (Figure 2). The quantitative vectorcardiogram analysis of $4634 \mathrm{R}$-waves yielded 113 false negative (Sensitivity $=97.6 \%$ ) and 76 false positive (Specificity: $98.4 \%$ ) events.

The reconstructed cine CMR images were free of visible trigger-related artefacts, and image quality was sufficient to quantitate both left and right ventricular volumes, ejection fraction, aortic and pulmonary blood flow and regurgitant fractions in all volunteers (Table 1, Figures 3-5).

One volunteer had difficulty with breath-holding and a ventricular ectopy, causing mild gating artefacts, which, however, did not affect quantitative analysis. In 2 other cases, image quality was slightly impaired by signal voids caused by focal RF field nonuniformities (Figure 3), but no case had level 3 artefacts precluding image analysis and volumetric quantitation. Mean image quality score was 0.9 (Table 1 ), indicating very good image quality overall. Mean left ventricular ejection fraction was $56.4 \%$ and mean right ventricular ejection fraction was $51.4 \%$ (Table 1 ).

Table 1. Quantitative volumetric analysis of 7T cardiovascular magnetic resonance (CMR) data sets for left and right ventricular volumes, and aortic and pulmonary flows.

\begin{tabular}{|c|c|c|c|c|c|c|c|c|c|c|c|c|}
\hline Subject & $\begin{array}{c}\text { LVEDV } \\
\mathrm{mL}\end{array}$ & $\begin{array}{c}\text { LVESV } \\
\text { mL }\end{array}$ & $\mathrm{SV} \mathbf{m L}$ & LVEF\% & Mass $\mathrm{g}$ & $\begin{array}{c}\text { RV-EDV } \\
\text { mL }\end{array}$ & $\begin{array}{c}\text { RV-ESV } \\
\text { mL }\end{array}$ & $\begin{array}{c}\text { RV-SV } \\
\text { mL }\end{array}$ & $\begin{array}{c}\text { RVEF } \\
\%\end{array}$ & $\begin{array}{c}\text { AO-SV } \\
\text { mL }\end{array}$ & $\begin{array}{c}\text { PA-SV } \\
\text { mL }\end{array}$ & $\begin{array}{c}\text { IQ } \\
\text { Score }\end{array}$ \\
\hline 1 & 146 & 72 & 74 & 51 & 123 & 167 & 85 & 82 & 49 & 117 & 133 & 1 \\
\hline 2 & 133 & 58 & 75 & 57 & 94 & 136 & 77 & 60 & 44 & 102 & 90 & 0 \\
\hline 3 & 120 & 60 & 59 & 60 & 124 & 134 & 71 & 63 & 47 & 50 & 56 & 1 \\
\hline 4 & 194 & 94 & 100 & 51 & 153 & 205 & 88 & 117 & 57 & 117 & 122 & 1 \\
\hline 5 & 172 & 77 & 96 & 56 & 128 & 195 & 87 & 108 & 55 & 105 & 116 & 1 \\
\hline 6 & 168 & 74 & 94 & 56 & 98 & 148 & 48 & 90 & 61 & 106 & 116 & 0 \\
\hline 7 & 106 & 45 & 61 & 57 & 94 & 139 & 74 & 65 & 47 & 62 & 71 & 2 \\
\hline 8 & 203 & 81 & 122 & 60 & 144 & 200 & 113 & 87 & 44 & 124 & 112 & 1 \\
\hline 9 & 142 & 61 & 81 & 57 & 93 & 157 & 75 & 82 & 52 & 90 & 92 & 0 \\
\hline 10 & 181 & 75 & 106 & 59 & 143 & 183 & 77 & 106 & 58 & 106 & 103 & 1 \\
\hline mean & 156.5 & 69.7 & 86.8 & 56.4 & 119.4 & 166.4 & 79.5 & 86 & 51.4 & 97.9 & 101.1 & 0.9 \\
\hline
\end{tabular}

LVEDV = left ventricular end diastolic volume, LVESV = left ventricular end systolic volume, LVEF = left ventricular ejection fraction, RVEDV = right ventricular end diastolic volume, RVESV = right ventricular end systolic volume, RVEF = right ventricular ejection fraction, $\mathrm{AoSV}=$ aortic stroke volume, $\mathrm{PASv}=$ pulmonary stroke volume, $\mathrm{IQ}=$ image quality artefact score where $0=$ no artefact, $3=$ severe artefact. 


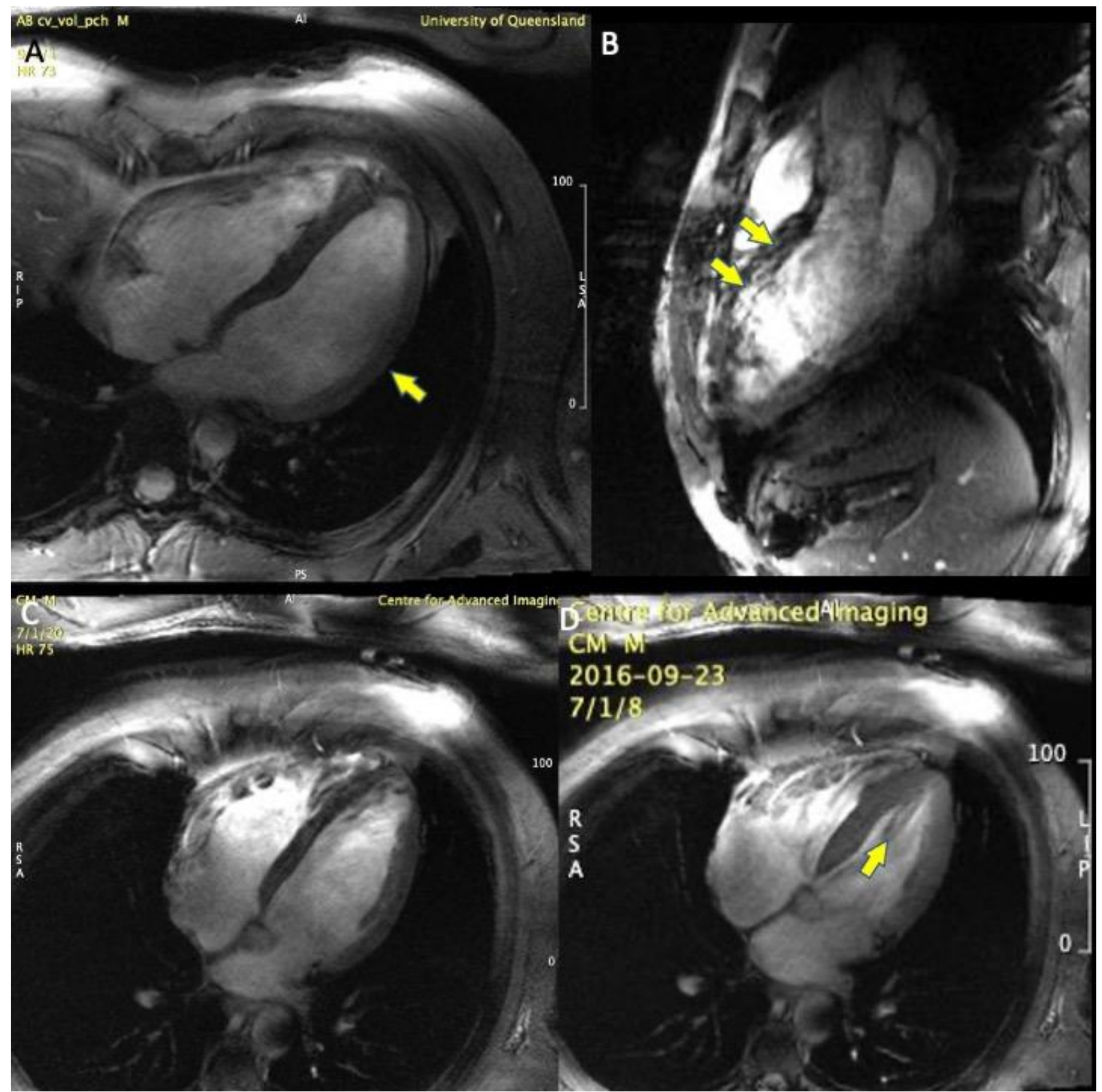

Figure 3. Image Quality of 7 T MRI with Vectorcardiogram gating. (A). 4 chamber view in diastole, showing good image quality, with mild signal dropout at the lateral left ventricular myocardial wall, yellow arrow (image quality score $=1$ ). (B). 3-chamber view showing substantial artefacts from susceptibility and motion in the anteriorly placed structures of the right ventricular outflow tract and the anteroseptal myocardium yellow arrows (image quality score $=2$ ). $(\mathbf{C}, \mathbf{D})$ ). 4-chamber diastolic and systolic frames, note the tricuspid valve leaflets are clearly seen in the closed position, and the mild flow artefacts in the left ventricular cavity during ejection, yellow arrow (image quality score $=1$ ). 


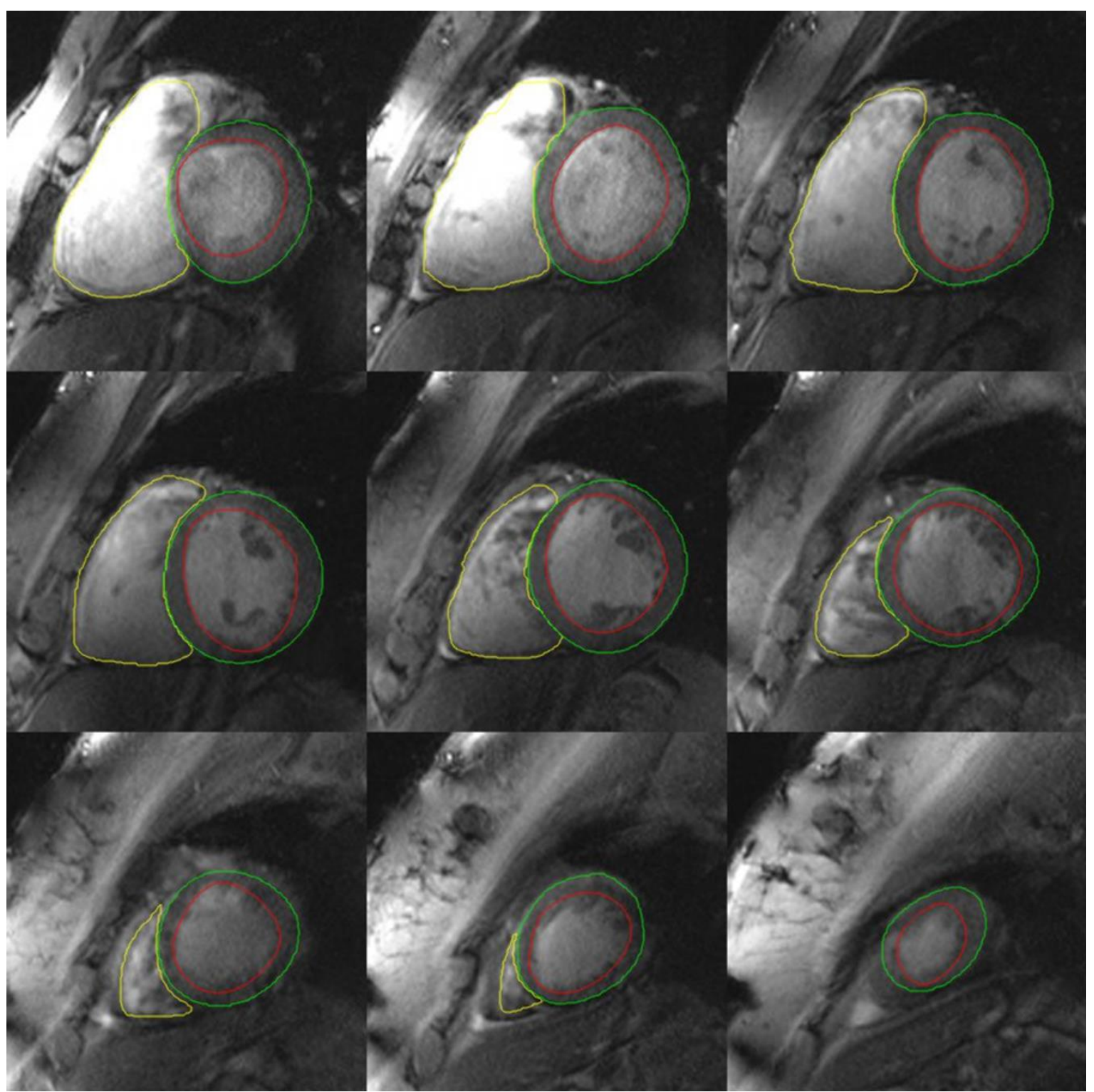

Figure 4. Quantitative volumetric analysis of left and right ventricular volumes from 7 T MRI. End-diastolic phase (red endocardial contour, green epicardial contour, yellow right ventricular contour). Note in the basal (top) slices there is bright signal in the right ventricular cavity due to coil inhomogeneity and proximity to the surface coil.

There was excellent correlation between Aortic and Pulmonary stroke volumes, correlation coefficient $\mathrm{R}=0.92$. There was good correlation between left ventricular stroke volume and aortic stroke volume, $\mathrm{R}=0.78$, with a bias of $-11 \mathrm{~mL}$ lower left ventricular stroke volume on Bland-Altman analysis, $p=0.03$, see Figure 5 . 


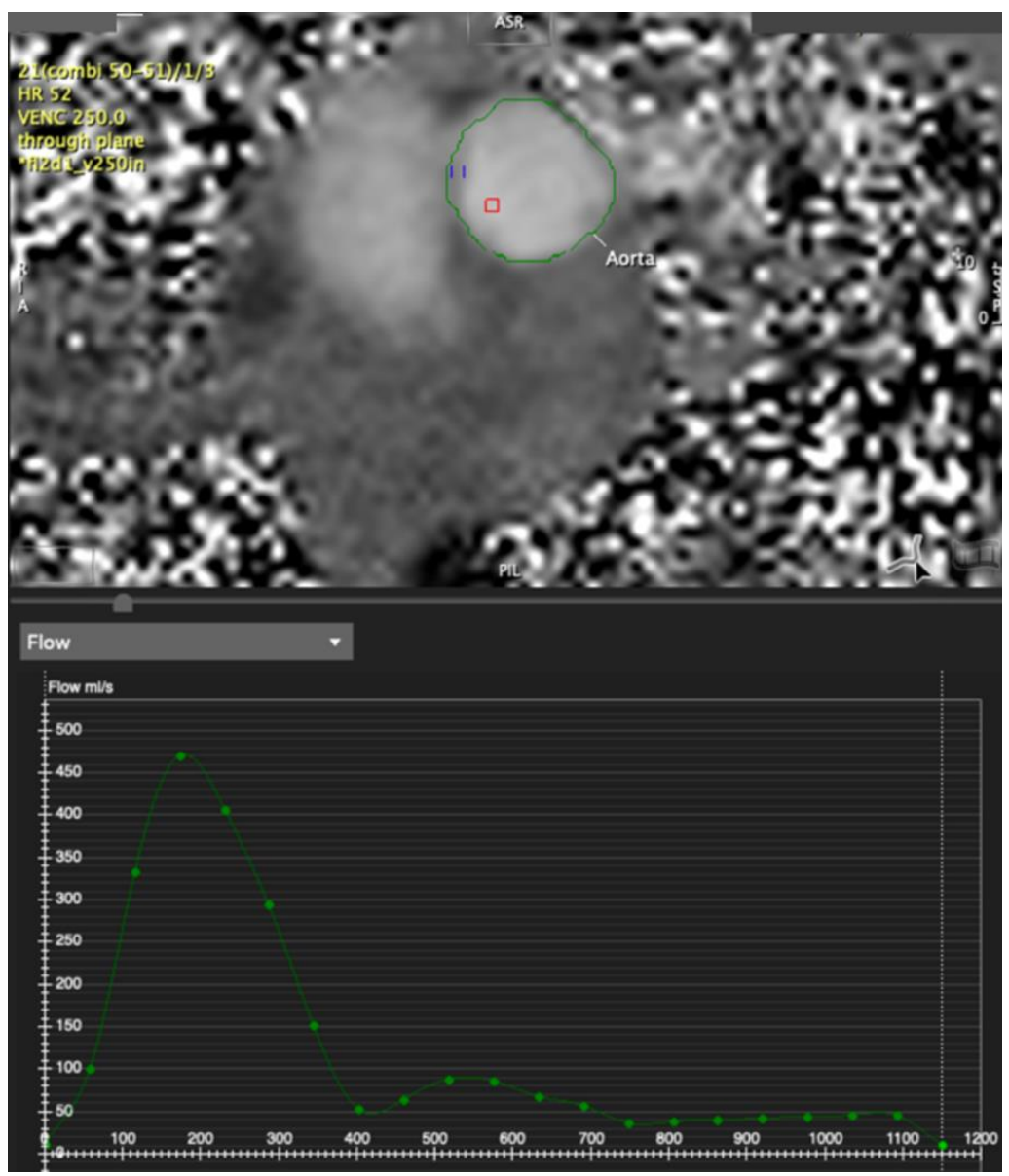

Figure 5. Aortic phase contrast flow imaging; upper panel phase image in the ascending aorta showing a very clean signal with sharp borders allowing ease of tracing along the aortic border. Lower panel: quantitative flow-time curve (processed with Circle Cvi42 software).

\section{Discussion}

Vectorcardiogram-triggered imaging, employing an out-of-magnet learning phase, overcame the magneto-hydrodynamic effect on ECG waveforms, allowing acquisition of high quality ECG-gated CMR at 7T with effective triggering for retrospectively segmented k-space CMR acquisitions.

Electrocardiographic signals are affected by the MHD effect due to the interaction between the blood, a ferromagnetic conductive fluid, and the surrounding magnetic field [4-8]. This interaction causes an electric field distortion, which is superimposed on the heart's intrinsic depolarization, thereby corrupting the signal transferred to cutaneous ECG electrodes. Importantly, the MHD effect is proportional to field strength (B0) and is substantially accentuated at ultra-high field. In addition, the MHD effect is augmented during cardiac systole at the time of maximal aortic blood flow and hence predominantly affects the QRS and T waves. This can cause inaccuracies in detecting the QRS complex and may result in impaired cardiac synchronized imaging. Including a sufficiently long VCG learning phase outside of the magnet bore, where the MHD effect is negligible, enabled this problem to be overcome $[8,10]$, resulted in effective R-wave recognition and successful cardiac synchronized cine imaging at 7T.

The ECG triggering approach used in this work provided the robustnes required for this feasibilty study. However, and as expected from the significant MHD related distortions of the ECG signal, triggering sensitivity and specificity were slightly reduced compared to using a similar VCG based approach at 1.5T. 
Alternative to the conventional ECG approach, a variety of other methods have been recently proposed, which can be used to tackle and improve triggering results at ultra-high field. Independent component analysis for example might have the potential to support and improve VCG based algorithms in detecting the R-wave [11]. Acoustic triggering detects the first heart tone instead of the R-wave, rendering the triggering unaffected by the MHD effect [12]. Similarly, ultrasound based triggering [13], pilot-tone navigation [14] or self-navigation approaches [15] may provide viable alternatives to conventional ECG triggering in ultra-high field environments.

We found that VCG-gated 7T CMR was feasible in healthy volunteers and provided clinically acceptable image quality enabling quantitation of left and right ventricular volumes and systolic function, and aorto-pulmonary vascular flow stroke volumes. Minimal artefacts due to the coil inhomogeneity, off-resonance, motion or a combination of these effects were observed; however, these did not interfere with interpretation and volumetric quantitation (Figure 2). The coil inhomogeneity made the right ventricle noticeably more difficult to contour on 7T images than on standard 1.5T and 3T images, with brighter signal anteriorly towards the surface coil and variable signal reduction in posterior myocardial wall (Figure 2, arrow). In addition, the use of spoiled gradient echo FLASH imaging, rather than steady-state free precession (SSFP) which is known to have improved tissue-blood contrast, made ventricular contouring more challenging than standard 1.5/3.0T images. However the gradient echo contrast is improved at 7T compared to 3T. SSFP imaging at 7T has been previously reported by the Oxford group using acoustic gating [5], however the transmit-receive cardiac coil used in our experiments did not allow for SSFP imaging due to exceeding SAR limitations. Parallel transmission coil technology may help to surmount this challenge to allow SSFP imaging at 7T [2].

The small negative bias of lower left ventricular stroke volume compared to the aortic stroke volume calculated from phase contrast imaging can be explained by the use of gradient echo FLASH imaging combined with the analysis technique of compacted myocardial contours in these healthy volunteers without valvular dysfunction. The reduced tissue-blood contrast of FLASH imaging compared to SSFP requires "smooth" endocardial contours which ignore trabeculations and papillary muscles [9], thus measuring a larger end-systolic volume and lower stroke volume. This accounts for the small negative bias of stroke volume measured by ventricular contours as compared to phase contrast imaging.

Spatial resolution was improved at 7T compared to standard CMR imaging. VCG triggering allowed cardiac synchronized ultra-high field imaging at a slice thickness of $4 \mathrm{~mm}$ with an isotropic in-plane resolution of $1.0 \mathrm{~mm}$, in comparison to a slice thickness of 6 to $8 \mathrm{~mm}$ and in-plane voxel resolution of 1.2 to $2.0 \mathrm{~mm}$ which is common at lower field strengths. Increased resolution may offer advantages in terms of the imaging of thin structures, such as the right ventricular free wall or subvalvular apparatus. SSFP imaging is the clinical standard for CMR imaging and would bring improvements in image quality and tissue-blood borders for volumetric quantitation. However, as discussed above, the use of SSFP was limited by SAR constraints arising from the current coil technology and the need for increased RF power to achieve the same flip angle.

The improved resolution, and reduction in eddy currents at high field strength with gradient shielding, also allowed for very crisp and high quality 2D flow imaging; this has the potential to improve the quantitation of valvular lesions such as aortic and pulmonary stroke volumes and assessment of regurgitation, for which CMR is emerging as the reference standard with improved reproducibility over echocardiographic Doppler-based assessments [16-18]. Gradient echo blood-tissue contrast is improved at 7T compared to standard field strengths, as seen in Figures 2 and 3. In addition, the increased signal and accurate ECG gating may also allow 4-Dimensional flow (4Dflow) at 7T.

Ideal candidates for imaging at 7T are those in sinus rhythm, able to breath-hold, and with no relative contraindications to MRI. 


\section{Limitations}

$\mathrm{B}_{1}+$ shimming was not conducted in order to facilitate clinically acceptable examination times for cardiac chamber quantification. The use of $B_{1}+$ shimming, which would be available through parallel transmission techniques, could be beneficial. However, $\mathrm{B}_{1}+$ shimming has implications for signal absorption rate (SAR) management and safety and was beyond the scope of this experiment. Subjects with arrhythmias such as atrial fibrillation were excluded, thus the performance of the VCG-gating algorithm in patients with arrhythmias is not known.

\section{Conclusions}

Reliable cardiac VCG triggering is feasible in healthy volunteers at ultra-high field, utilizing a state-of-the-art three-lead trigger device with out-of-magnet learning phase, overcoming signal distortion from the MHD effect. This provided sufficient image quality for quantitative analysis. Other ultra-high-field imaging applications such as $4 \mathrm{D}$ flow and human brain functional MRI with physiologic noise correction may benefit from this method of ECG triggering.

Author Contributions: Conceptualization, C.H.-C., M.B., G.G.; methodology, C.H.-C., M.B., G.G., D.S., K.O.; data collection, A.A.N., W.C., S.F., C.H.-C., G.G.; formal analysis, C.H.-C.; writingoriginal draft preparation, C.H.-C.; writing-review and editing, M.B., G.G., D.S., K.O.; supervision, C.H.-C., M.B., G.G.; funding acquisition, C.H.-C. All authors have read and agreed to the published version of the manuscript.

Funding: This work was supported by UQ Academic Title Holder Research Fund.

Institutional Review Board Statement: The study was conducted according to the guidelines of the Declaration of Helsinki and approved by thes Ethics Review Board of The University of Queensland; approval number UQ 200500050.

Informed Consent Statement: Informed consent was obtained from all subjects involved in the study.

Data Availability Statement: Additional data are available from the Lead/Corresponding author upon reasonable request.

Acknowledgments: The authors acknowledge the facilities and the scientific and technical assistance of the National Imaging Facility at the Centre for Advanced Imaging, University of Queensland, Wendy Strugnell FSMRT, the electrophysiology scientists of The Prince Charles Hospital, and funding from the UQ Academic Title Holders grant.

Conflicts of Interest: The authors declare no conflict of interest.

\section{References}

1. Hamilton-Craig, C.; Slaughter, R.E.; Maki, J. Cardiovascular magnetic resonance from basics to clinical applications. Appl. Radiol. 2010, 39, 43-52.

2. Gutberlet, M.; Schwinge, K.; Freyhardt, P.; Spors, B.; Grothoff, M.; Denecke, T.; Lüdemann, L.; Noeske, R.; Niendorf, T.; Felix, R. Influence of high magnetic field strengths and parallel acquisition strategies on image quality in cardiac 2D CINE magnetic resonance imaging: Comparison of 1.5 T vs. 3.0 T. Eur. Radiol. 2005, 15, 1586-1597. [CrossRef] [PubMed]

3. Kraff, O.; Fischer, A.; Nagel, A.; Mönninghoff, C.; Ladd, M.E. MRI at 7 tesla and above: Demonstrated and potential capabilities. J. Magn. Reson. Imaging 2015, 41, 13-33. [CrossRef] [PubMed]

4. Sievers, B.; Wiesner, M.; Kiria, N.; Speiser, U.; Schoen, S.; Strasser, R.H. Influence of the trigger technique on ventricular function measurements using 3-Tesla magnetic resonance imaging: Comparison of ECG versus pulse wave triggering. Acta Radiol. 2011, 52, 385-392. [CrossRef] [PubMed]

5. $\quad$ Suttie, J.J.; DelaBarre, L.; Pitcher, A.; Van De Moortele, P.F.; Dass, S.; Snyder, C.; Francis, J.M.; Metzger, G.; Weale, P.; Ugurbil, K.; et al. 7 Tesla (T) human cardiovascular magnetic resonance imaging using FLASH and SSFP to assess cardiac function: Validation against 1.5 T and 3 T. NMR Biomed. 2011, 25, 27-34. [CrossRef]

6. Stäb, D.; Al Najjar, A.; O’Brien, K.; Strugnell, W.; Richer, J.; Rieger, J.; Niendorf, T.; Barth, M. Cardiac Magnetic Resonance Imaging at 7 Tesla. J. Vis. Exp. 2019, 143, e55853. [CrossRef]

7. Frank, M.R.J. Method for Identifying an R-Wave in an ECG Signal, ECG Measuring Device and Magnetic Resonance Scanner. U.S. Patent No. 8,332,023, 11 December 2012. 
8. Stäb, D.; Roessler, J.; O’Brien, K.; Hamilton-Craig, C.; Barth, M. ECG Triggering in Ultra-High Field Cardiovascular MRI. Tomography 2016, 2, 167-174. [CrossRef] [PubMed]

9. Moody, W.E.; Hudsmith, L.E.; Holloway, B.; Treibel, T.A.; Davies, R.; Kozor, R.; Hamilton-Craig, C.; Edwards, N.C.; Bradlow, W.M.; Moon, J.C.; et al. Variation in cardiovascular magnetic resonance myocardial contouring: Insights from an international survey. J. Magn. Reson. Imaging 2019, 50, 1336-1338. [CrossRef] [PubMed]

10. Knesewitsch, T.; Meierhofer, C.; Rieger, H.; Rößler, J.; Frank, M.; Martinoff, S.; Hess, J.; Stern, H.; Fratz, S. Demonstration of value of optimizing ECG triggering for cardiovascular magnetic resonance in patients with congenital heart disease. J. Cardiovasc. Magn. Reson. 2013, 15, 3. [CrossRef] [PubMed]

11. Krug, J.W.; Rose, G.; Clifford, G.D.; Oster, J. ECG-based gating in ultra high field cardiovascular magnetic resonance using an independent component analysis approach. J. Cardiovasc. Magn. Reson. 2013, 15, 104. [CrossRef] [PubMed]

12. Frauenrath, T.; Hezel, F.; Renz, W.; d'Orth, T.G.; Dieringer, M.; von Knobelsdorff-Brenkenhoff, F.; Prothmann, M.; Schulz, M.J.; Niendorf, T. Acoustic cardiac triggering: A practical solution for synchronization and gating of cardiovascular magnetic resonance at 7 Tesla. J. Cardiovasc. Magn. Reson. 2010, 12, 67. [CrossRef] [PubMed]

13. Kording, F.; Ruprecht, C.; Schoennagel, B.; Fehrs, K.; Yamamura, J.; Adam, G.; Goebel, J.; Nassenstein, K.; Maderwald, S.; Quick, H.; et al. Doppler ultrasound triggering for cardiac MRI at 7T. Magn. Reson. Med. 2018, 80, 239-247. [CrossRef] [PubMed]

14. Ludwig, J.; Speier, P.; Seifert, F.; Schaeffter, T.; Kolbitsch, C. Pilot tone-based motion correction for prospective respiratory compensated cardiac cine MRI. Magn. Reson. Med. 2021, 85, 2403-2416. [CrossRef] [PubMed]

15. Kolbitsch, C.; Prieto, C.; Schaeffter, T. Cardiac functional assessment without electrocardiogram using physiological selfnavigation. Magn. Reson. Med. 2014, 71, 942-954. [CrossRef] [PubMed]

16. Cawley, P.J.; Hamilton-Craig, C.; Owens, D.S.; Krieger, E.V.; Strugnell, W.E.; Mitsumori, L.; D’Jang, C.L.; Schwaegler, R.G.; Nguyen, K.Q.; Nguyen, B.; et al. Prospective Comparison of Valve Regurgitation Quantitation by Cardiac Magnetic Resonance Imaging and Transthoracic Echocardiography. Circ. Cardiovasc. Imaging 2013, 6, 48-57. [CrossRef] [PubMed]

17. Krieger, E.V.; Lee, J.; Branch, K.; Hamilton-Craig, C. Quantitation of mitral regurgitation with cardiac magnetic resonance imaging: A systematic review. Heart 2016, 102, 1864-1870. [CrossRef] [PubMed]

18. Lee, J.C.; Branch, K.; Hamilton-Craig, C.; Krieger, E.V. Evaluation of aortic regurgitation with cardiac magnetic resonance imaging: A systematic review. Heart 2017, 104, 103-110. [CrossRef] [PubMed] 